Published in final edited form as:

Pediatr Clin North Am. 2015 February ; 62(1): 61-73. doi:10.1016/j.pcl.2014.09.006.

\title{
Treatment of Pediatric Acute Lymphoblastic Leukemia
}

\author{
Stacy L. Cooper, MDa and Patrick A. Brown, MD ${ }^{b,{ }^{*}}$ \\ aPediatric Hematology/Oncology, Johns Hopkins/National Institutes of Health, Bloomberg 11379, \\ 1800 Orleans Street, Baltimore, MD 21287, USA \\ bediatric Leukemia Program, Sidney Kimmel Comprehensive Cancer Center, Johns Hopkins \\ University School of Medicine, 1650 Orleans Street, CRB1 Room 2M49, Baltimore, MD 21231, \\ USA
}

\section{Keywords}

Acute lymphoblastic leukemia; Leukemia treatment; Risk-based stratification

\section{INTRODUCTION}

Acute lymphoblastic leukemia (ALL) is the most common cancer diagnosed in children. It has an overall survival of approximately $80 \%$, with certain subsets experiencing greater than $98 \%$ cure rate $^{1}$

Incremental advances in therapy have led to marked improvements in survival since it was first treated, with these advances highlighting the importance of clinical trials through cooperative multicenter groups (Table 1). Childhood ALL also often serves as the paradigm for risk-based therapy, whereby stratification of treatment intensity is based on risk of treatment failure (Fig. 1).

\section{RISK STRATIFICATION OF NEWLY DIAGNOSED ACUTE LYMPHOBLASTIC LEUKEMIA}

One of the hallmarks of the treatment of childhood ALL is the reliance on risk-based stratification. By identifying the features that have been shown to affect prognosis, patients can be classified into groups based on risk of treatment failure. Those with favorable features can be treated with less toxic regimens, whereas more aggressive regimens are reserved for those with more high-risk disease.

It is therefore paramount to identify those features shown to consistently affect prognosis and, thus, influence treatment. Several clinical characteristics have been shown to aid in this classification, including age and white blood cell count (WBC) at presentation, together referred to as the National Cancer Institute (NCI) criteria. Age between 1 and 10 years is a standard risk feature, with more aggressive disease seen in infants and those older than 10

(C) 2015 Elsevier Inc. All rights reserved.

*Corresponding author. pbrown2@jhmi.edu. 
years. In part this is due to the higher rate of favorable cytogenetics in those aged 1 to 10 years. ${ }^{2}$ The initial WBC at presentation also has been directly associated with increased risk, with high risk noted with WBC greater than $50,000 / \mu \mathrm{L}$. Of importance is that this is a continuous function, but for practical purposes this threshold has been chosen as a useful categorical cut-off. The application of the NCI criteria results in those aged 1 to 10 years with initial WBC less than 50,000/ $\mu \mathrm{L}$ classified as standard risk, with those not meeting those parameters classified as high risk.

Sanctuary sites are extramedullary anatomic locations that have historically been difficult to penetrate with systemic chemotherapy, and involvement of these sites at initial diagnosis has thus also been considered a high-risk feature. Approximately 3\% of patients will demonstrate overt central nervous system (CNS) disease at diagnosis, defined either as a diagnostic lumbar puncture with the presence of leukemic blasts on cytospin and greater than 5 leukocytes/ $\mu \mathrm{L}$, or clinical evidence of CNS involvement (such as a cranial nerve palsy). Approximately $2 \%$ of boys with newly diagnosed ALL will present with testicular involvement, usually presenting with an enlarged, nonpainful testis. Leukemic involvement of the CNS or testis precludes a classification of standard risk in most treatment schemas.

Patients treated with corticosteroids before their complete diagnostic workup are also considered as high risk, as the tremendous efficacy of steroids to treat ALL may underestimate initial WBC and involvement of sanctuary sites, and limit confidence in staging.

The characteristics of the leukemia cells themselves can also be used to determine which patients are at higher risk. The immunophenotype describes the leukemic cells in terms of the proteins that are expressed, and whether these are more similar to cells that would eventually become B lymphocytes or T lymphocytes. This determination has also been shown to affect prognosis. At approximately 80\%, most pediatric patients with ALL have Bprecursor immunophenotype (Bp ALL), which encompasses a broad range of patients, including many of the lowest-risk patients with childhood ALL. Conversely, those with Tcell immunophenotype comprise approximately $10 \%$ to $15 \%$ of pediatric ALL, and have historically been associated with a lower cure rate. However, identification of these patients and treatment with more aggressive regiments has led to survival rates that approach that of Bp ALL, ${ }^{1,3}$ with the possible exception of early T-precursor (ETP) ALL, a particular subset of T-cell ALL that has been associated with a poor prognosis in some studies. ${ }^{4}$ Additional rare immunophenotypic groups include those acute leukemias of mixed lineage, which occur in less than 5\% of pediatric acute leukemias. These groups include undifferentiated acute leukemias that cannot be sufficiently characterized as either lymphoid or myeloid in origin, as well as those biphenotypic lineages that include markers of both myeloid and lymphoid origins and/or both B-cell and T-cell origins. These ambiguous immunophenotypes are often inconsistently defined but, regardless of exact classification, are associated with a poorer prognosis. ${ }^{5}$

Recurrent cytogenetic abnormalities in the leukemic blasts allow a molecular classification of risk, with certain markers shown to be associated with favorable or unfavorable outcomes. The 2 most well-established favorable cytogenetic aberrations include high 
hyperdiploidy and the ETV6/RUNX1 translocation. High hyperdiploidy is seen in 20\% to $25 \%$ of cases of Bp ALL. It is defined as 51 to 65 chromosomes per cell or a DNA index of greater than 1.16, and is particularly favorable when associated with simultaneous trisomy 4 and $10 .{ }^{6}$ The ETV6/RUNX1 translocation (due to t[12;21], formerly TEL/AML1) is also seen in approximately $20 \%$ to $25 \%$ of cases of Bp ALL, and is associated with improved survival, including improved survival even after relapse. ${ }^{7}$ Both favorable subgroups occur in lower frequency in African Americans (sub-Saharan Africans), and in part accounts for the lower overall outcome in this population. ${ }^{8,9}$ Several unfavorable cytogenetic changes have also been identified. One feature strongly associated with poor outcome is hypodiploidy, defined as fewer than 44 chromosomes or a DNA index of less than 0.81. Additional cytogenetic changes associated with higher-risk ALL include BCR-ABL fusion of $\mathrm{t}(9 ; 22)$, known as the Philadelphia chromosome (seen in $3 \%$ of pediatric ALL), MLL rearrangements involving 11q23 (seen in 5\% of pediatric ALL, often infants and adolescents), and, most recently identified, intrachromosomal amplification of chromosome 21 (iAMP21, seen in $1 \%-2 \%$ of Bp ALL). ${ }^{10}$

In addition to these features that are used to inform prognosis, the response to the initial therapy has emerged as a particularly powerful independent predictor. Traditionally a complete remission has been defined as less than 5\% detectable blasts on microscopic morphology at the end of induction. Induction failure is seen in approximately $3 \%$ to $5 \%$ of children with newly diagnosed ALL and portends a very poor prognosis, with an overall survival of approximately $33 \%$. It is most closely associated with patients with T-cell immunophenotype, Bp immunophenotype with a high presenting leukocyte count, $M L L$ rearrangement, Philadelphia chromosome, or older age. ${ }^{11}$

Evaluation of the bone marrow by microscopy is often relatively insensitive, and has been shown to be complemented and, in part, displaced, by evaluation of minimum residual disease (MRD). This technique uses flow cytometry or the polymerase chain reaction (PCR) to assess for disease at a significantly lower limit of detection (1 leukemic blast in 10,000100,000 cells). Evaluation of bone marrow MRD at the end of induction has proved to be an independent factor predicting outcome, and has also been shown to be useful in the peripheral blood as early as day 8 of therapy. End-induction MRD has been established in the risk stratification of Bp ALL patients ${ }^{12}$ while studies using MRD to adjust the treatment of T-cell ALL are currently ongoing but also promising.

The application of these risk factors is operationalized in various methods by the different cooperative groups of pediatric oncology specialists. One group, the Children's Oncology Group (COG), utilizes a combination of the NCI criteria in addition to cytogenetics and response to therapy. Other groups, such as the Berlin-Franklin-Münster (BFM) Group, rely almost solely on response to initial therapy using MRD thresholds, although with certain cytogenetic changes treated as high risk regardless of response to therapy (Box 1). ${ }^{13}$

Box 1

High-risk features in pediatric acute lymphocytic leukemia (ALL)

- Age less than 1 year old or greater than 10 years old 
- Initial white blood cell count greater than $50,000 / \mu \mathrm{L}$

- Central nervous system involvement

- Testicular involvement

- Unfavorable cytogenetics (hypodiploidy, $\mathrm{t}(9 ; 22), 11 \mathrm{q} 23$, iAMP21)

- Suboptimal induction response (induction failure or positive minimum residual disease)

\section{TREATMENT OF NEWLY DIAGNOSED ACUTE LYMPHOBLASTIC LEUKEMIA}

There are 4 major components of treatment of newly diagnosed ALL, reflecting a reliance on multidrug regimens to avoid development of resistance. Different blocks of chemotherapy have varying intensity depending on the group of patients at risk, with increasingly intensive regimens corresponding to more aggressive disease categories.

Remission induction is the first block of chemotherapy, lasting 4 to 6 weeks. Patients are usually admitted to the hospital for their initial treatment and workup, but once any complications have stabilized the patient may be discharged before the completion of this phase with close outpatient follow-up. The goal of this block of therapy is to induce a complete remission by its completion, with approximately $95 \%$ of all patients achieving this benchmark. Of those that do not achieve completion remission by the end of induction, half suffer induction failure and the remainder succumb to treatment-related mortality. For those with induction failure, an allogeneic bone marrow transplant is usually pursued, although there is no consensus standard of care regarding the chemotherapy used to achieve remission before transplant. ${ }^{11}$

The agents used during induction include vincristine, corticosteroids, and asparaginase, with most regimens adding an anthracycline (usually doxorubicin or daunorubicin). Both anthracyclines have been shown to have similar efficacy and toxicity in randomized trials. ${ }^{14}$ Certain groups spare the addition of anthracyclines to those lower-risk groups in an effort to decrease toxicity. The corticosteroid used is usually prednisone or dexamethasone, with dexamethasone demonstrating improved CNS penetration and decreased risk of relapse, but with increased incidence of toxicities, including avascular necrosis, infection, and reduction in linear growth. ${ }^{15}$ Several different agents for asparagine depletion exist as well, including PEG asparaginase and Erwinia asparaginase. PEG asparaginase has been modified by covalently attaching polyethylene glycol, which has been demonstrated to result in a longer half-life and decreased immunogenicity in comparison with native Escherichia coli Lasparaginase. Randomized trials have also shown superior efficacy of the pegylated formulation. ${ }^{16}$ Erwinia asparaginase is usually given to those patients who have experienced an allergic reaction to PEG asparaginase, and requires a more frequent administration schedule.

Remission induction is followed by consolidation, which aims to eradicate the submicroscopic residual disease that remains after a complete remission is obtained. Lasting 
approximately 6 to 9 months, it varies in length and intensity among different protocols, with those patients with higher-risk disease receiving longer and more intensive consolidation regimens. ${ }^{17}$ Consolidation is usually administered on an outpatient basis, although there are protocols with more aggressive regimens that require inpatient care. This phase of chemotherapy involves combinations of different chemotherapeutic agents to maximize synergy and minimize drug resistance, often including agents not used in the initial remission induction, such as mercaptopurine, thioguanine, methotrexate, cyclophosphamide, etoposide, and cytarabine.

Maintenance chemotherapy is the final, and longest, stage of treatment in childhood ALL. A much less intensive regimen than the prior chemotherapy, the prolonged maintenance phase has been demonstrated to lower the risk of relapse once remission has been established. It usually lasts at least 2 years (extended to 3 years for boys in some protocols), is administered on an outpatient basis, and typically is associated with less disruptive toxicity. The cornerstone of maintenance therapy is antimetabolite therapy with methotrexate and mercaptopurine, both available in oral formulations, making strict adherence crucial. ${ }^{18}$ Furthermore, emerging evidence regarding the pharmacogenomics of these drugs underscores the importance of interindividual differences in metabolism. For example, genotypic polymorphisms in the enzyme thiopurine methyltransferase are associated with increased myelosuppression and other toxicities, whereas other polymorphisms confer a "hypermetabolizer" state, with decreased levels of the active metabolite. ${ }^{19}$ Understanding these differences in metabolism is particularly important because studies have shown that the degree of myelosuppression correlates with relapse risk. ${ }^{20,21}$ Accordingly, many protocols include guidelines for dose adjustments to assist in achieving the goal of balancing the risks of inadequate myelosuppression with the risks of severe pancytopenia (infection, bleeding, and so forth). Some regimens also include monthly vincristine and steroids, although the evidence for additional benefit is unclear. ${ }^{22}$

The fourth component of the treatment of ALL is therapy directed against the CNS. This approach includes both treatment of patients with clinical CNS disease at diagnosis and prophylaxis for patients with subclinical disease. The importance of this component was clearly demonstrated before the 1970s, when treatment lacked this component. Although bone marrow remission could be achieved using systemic chemotherapy, most children eventually developed CNS relapse in the absence of specific therapy directed toward this sanctuary site. ${ }^{23}$

There are several methods of achieving the goal of eradication of disease from the CNS, including direct intrathecal administration of chemotherapy, systemic administration of chemotherapy able to penetrate the blood-brain barrier, and cranial radiation. All treatment plans include intrathecal administration of chemotherapy beginning during remission induction. Some protocols include intrathecal treatment throughout therapy, whereas others do not include it in maintenance. Options for intrathecal chemotherapy include including intrathecal methotrexate or a combination of intrathecal methotrexate, cytarabine, and hydrocortisone (known as triple intrathecal). Studies have shown no definitive difference in overall or event-free survival between the two, although some evidence points to decreased frequency of CNS relapse with the use of triple intrathecal therapy. ${ }^{24}$ Systemically 
administered chemotherapy with CNS effects includes dexamethasone, high-dose methotrexate, cytarabine, and asparaginase.

Given the risk of toxicity of cranial radiation, manifesting primarily as intellectual disability (particularly with younger patients) and as second malignant neoplasms, its utilization has been progressively declining. Many protocols reserve its use for only those at highest risk of CNS relapse while some institutions defer its use altogether. For those patients with overt CNS disease at presentation, several small studies have shown that by increasing the intensity of the intrathecal and systemic chemotherapy, cranial irradiation can also be deferred for these patients. However, larger studies are needed to confirm this strategy. ${ }^{25,26}$

The role of allogeneic hematopoietic stem cell transplant (HSCT) in first remission of ALL is not yet well defined, and is a controversial topic. Broadly speaking, HSCT is considered for those patients with the very highest risk of relapse and/or treatment failure, which has been most closely associated with those patients demonstrating hypodiploidy or induction failure. General tenets of HSCT for ALL include the use of total body irradiation (TBI) in the preparative regimen, and improved outcomes for patients who undergo transplant after achieving MRD-negative disease status. The optimal donor has historically been a matched sibling, although advances with alternative donor sources are now also showing promise. ${ }^{27}$

\section{CONSIDERATIONS FOR PARTICULAR SUBGROUPS OF ACUTE LYMPHOBLASTIC LEUKEMIA}

Infants with ALL represent a particularly high-risk subclass, with higher risks of both treatment failure and treatment complications. The highest rates of treatment failure are seen in infants diagnosed before 6 months of age, those with high initial WBC count, or those with $M L L$ gene rearrangements (which occur in 70\%-80\% of infants diagnosed with ALL). Infant regimens often contain intensive chemotherapy not typically administered on other ALL protocols. In cases with $M L L$ rearrangement, the leukemic cells typically express high levels of FLT3, a tyrosine kinase oncogene; therefore, studies are currently ongoing to test the addition of a FLT3 inhibitor to conventional chemotherapy regimens. In addition, these patients have a high risk of treatment-related mortality, ${ }^{28}$ and thus induction often includes a 1-week prophase of single-agent steroid to "debulk" the initial leukemic burden before initiation of multi-agent chemotherapy. Similar to MRD evaluation, response to this steroid prophase has been shown to correlate with risk of treatment failure. ${ }^{29}$ Infants also have a particularly high rate of infectious complications and thus warrant aggressive supportive care, including broad-spectrum antimicrobial prophylaxis, use of growth factors, and inpatient management.

Adolescents have been shown to have lower rates of overall survival, in part owing to the association of an increased incidence of Philadelphia chromosome and T-cell immunophenotype. Therefore, most protocols will consider patients older than 10 years as high risk, with some protocols classifying those older than 13 years as "very high risk" based on age alone. Young adult patients also experience a higher rate of treatment-related morbidity and mortality, particularly secondary to infection, osteonecrosis, and 
thrombosis. ${ }^{30,31}$ Multiple studies have demonstrated the benefit of treating adolescent and young adult patients with ALL on pediatric-based protocols. ${ }^{32}$

Patients with T-cell ALL comprise approximately $10 \%$ of pediatric ALL and, compared with those with Bp-ALL, have historically experienced a worse prognosis. With more aggressive modern regimens, however, many patients with T-ALL have survival approaching that of Bp-ALL. Unfortunately, T-ALL patients continue to experience a lower risk of survival after relapse. Studies are currently ongoing regarding the addition of nelarabine, a purine nucleoside analogue that appears to be particularly cytotoxic to $\mathrm{T}$ cells, with promising results in the relapsed setting. 33

As discussed earlier, patients with mixed-lineage leukemia often represent a relatively recalcitrant subgroup. Although there is no well-defined consensus for the treatment of these patients, many groups recommend the combination of therapy directed at both lineages, and have shown that cure can potentially be achieved without the use of HSCT. ${ }^{34}$

Children with trisomy 21 (Down syndrome) have an increased risk of ALL, with a lower incidence of both favorable and unfavorable cytogenetics. These patients demonstrate a similar rate of relapse compared with patients without Down syndrome, but have an increased risk of treatment-related mortality, primarily secondary to infectious deaths. Therefore they are typically treated on specific protocols with enhanced supportive measures and more frequent use of leucovorin rescue to mitigate the toxic effects of methotrexate 35 (see the relevant article elsewhere in this issue for further details on Down syndrome and leukemia).

Chromosomal translocation of chromosomes 9 and 22, known as the Philadelphia chromosome and resulting in the fusion product BCR-ABL, occurs in approximately $3 \%$ of childhood ALL. Although these patients are classified as high risk, the introduction of imatinib, a tyrosine kinase inhibitor that targets the BCR-ABL fusion protein, has markedly improved the outcome of this disease. Addition of this agent to intensive, multidrug chemotherapy regimens has been shown to result in far superior event-free and overall survival in comparison with historical controls, and has rendered unclear the role of HSCT for these patients. ${ }^{36}$ Newer generations of tyrosine kinase inhibitors (such as dasatinib, nilotinib, and ponatinib) have been recently introduced with evidence of improved efficacy in adults.

\section{TREATMENT OF RELAPSED ACUTE LYMPHOBLASTIC LEUKEMIA}

Despite significant advances in treatment, approximately $15 \%$ to $20 \%$ of patients with ALL will suffer relapsed disease, the most common cause of treatment failure. With intensive therapy that may include HSCT, overall survival from relapsed ALL is approximately $40 \% .{ }^{37}$ Similar to those patients with newly diagnosed ALL, those with relapsed disease can be risk stratified. Length of first complete remission (CR1) and site of relapse have consistently been demonstrated to be the 2 most important prognostic factors in these cases. For patients with Bp-ALL, relapses within 18 months of diagnosis fare the worst, those occurring between 18 and 36 months after diagnosis have an intermediate prognosis, and 
late relapses that occur more than 3 years from diagnosis have the best prognosis, with up to a $50 \%$ event-free survival. ${ }^{38}$

Site of relapse is the other salient risk feature to consider in relapsed disease, with isolated marrow relapses as the most common site, occurring in $50 \%$ to $60 \%$ of cases. The remainder comprises isolated CNS disease in approximately $20 \%$, isolated testicular disease in approximately $5 \%$, and a combination of marrow and extramedullary disease in the remainder. Isolated extramedullary relapses have the best prognosis, with the worst outcomes seen in isolated marrow relapses. Those with combined marrow and extramedullary involvement have an intermediate prognosis. ${ }^{39}$

The risk group at initial diagnosis has been shown to also play a role in the relapse setting, with increased survival in those initially classified as standard risk compared with those at high risk. This finding holds particularly true when considering those with T-cell immunophenotype, who experience a particularly poor prognosis after relapse. As with patients with newly diagnosed ALL, the response to reinduction chemotherapy has prognostic significance. Those with persistent morphologic disease after the first cycle of reinduction chemotherapy have an especially poor prognosis, and those with a morphologic remission but continued detectable minimal residual disease have a worse outcome than those with MRD-negative disease after reinduction. ${ }^{40,41}$

The application of cytogenetic abnormalities in the risk stratification of relapsed ALL has been limited, although a few have been shown to be informative. For example, those with relapsed disease who demonstrate ETV6-RUNX1 mutations have a relatively favorable prognosis, with an event-free survival of more than $80 \%$ if initial CR1 was at least 36 months. ${ }^{42}$ Conversely, blasts demonstrating TP53 mutations show a particularly poor prognosis. $^{43}$

Reinduction chemotherapy after first relapse is successful at inducing complete remission in $65 \%$ to $85 \%$. $^{44}$ The chemotherapy regimens used vary by institution and protocol, but is often the same 4-drug induction used at initial diagnosis, consisting of vincristine, steroids, asparaginase, and an anthracycline. Clinical trials are currently ongoing to evaluate the addition of novel agents for reinduction chemotherapy but, given the lack of clear data, no consensus yet exists. Once a second complete remission (CR2) has been obtained, postremission treatment varies by risk. Those patients with T-cell immunophenotype (regardless of duration of CR1) or Bp-ALL early relapsed marrow disease are usually treated with HSCT. Those with Bp-ALL late marrow relapses can often be cured with chemotherapy alone, and this risk stratification in part relies on the use of detection of MRD at the end of reinduction. ${ }^{45}$

Patients with isolated CNS relapse usually receive a combination of chemotherapy and cranial radiation, with chemotherapy administered first to prevent an overt marrow relapse. Craniospinal radiation has not been shown to have increased efficacy, and therefore the addition of spinal radiation has largely been abandoned in contemporary trials. For those isolated CNS Bp-ALL relapses occurring more than 18 months from diagnosis, survival rates of $70 \%$ can be achieved with chemoradiation alone, and thus HSCT is usually not 
required. For those with early isolated CNS relapses and/or T-cell immunophenotype, prognosis is worse, and HSCT is often pursued, although no clear data exist regarding whether HSCT leads to superior outcomes. Treatment of isolated testicular relapse also depends on duration of CR1, with worse outcomes for those patients experiencing an isolated testicular relapse while still receiving upfront therapy. Therapy for testicular relapse usually consists of intensive reinduction chemotherapy (often including high-dose methotrexate) followed by testicular radiation or orchiectomy if complete remission is not achieved. ${ }^{46}$

Therapy for second and subsequent relapses is varied and without clear evidence-based guidance. Long-term survival is generally poor for these patients. For those receiving chemotherapy alone for their first relapse, HSCT once third remission (CR3) has been achieved is the standard therapy. For those who received HSCT in CR2, a select population of patients may be considered for a second HSCT. Donor leukocyte infusions are usually unsuccessful in achieving durable remissions in relapsed ALL after HSCT, particularly when used as monotherapy. ${ }^{47}$

\section{NOVEL AGENTS IN THE TREATMENT OF ACUTE LYMPHOBLASTIC LEUKEMIA}

Current efforts in advancing the treatment of ALL focus on unique mechanisms that contrast with the nonspecific targeting of conventional chemotherapy. Immunotherapy is a broad and promising field that seeks to harness the power of the immune system to allow for a more targeted approach. Chimeric antigen receptors are one example of modified adoptive cell transfer whereby the patient's own cytotoxic T cells are genetically engineered to express an antibody to target leukemic antigens (often CD19), often enhanced by the inclusion of costimulatory binding regions that allow for improved cytotoxicity and duration of cells. ${ }^{48}$ Another example of immunotherapy is blinatumomab, a bispecific anti-CD19/CD3 molecule, which enhances cytotoxic killing by binding both a protein expressed on the leukemic blast (CD19) and one expressed on autologous T cells (CD3). ${ }^{49}$ Finally, moxetumomab is an antibody conjugate wherein a monoclonal antibody recognizing CD22 is fused with a fragment of the Pseudomonas exotoxin, to allow for direct cell killing. ${ }^{50}$ All 3 of these have shown promising results in early clinical trials, with larger studies under way. Another promising novel treatment strategy focuses on the epigenetic changes seen in leuke-mogenesis, with the use of histone deacetylase inhibitors (HDACi), such as vorinotstat. ${ }^{51}$ Finally, bortezomib is a proteasome inhibitor that interferes with natural killer $\mathrm{\kappa B}$ signaling and is able to enhance bcl-2 and bcl-x, rendering blasts more sensitive to apoptosis, particularly in combination with conventional chemotherapy agents. ${ }^{52}$

\section{TOXICITIES IN THE TREATMENT OF ACUTE LYMPHOBLASTIC LEUKEMIA}

Almost all chemotherapy agents cause myelosuppression, mucositis, and nausea/vomiting. Unique effects of common chemotherapeutic agents used in the treatment of ALL are listed in Box 2. 


\section{Box 2}

\section{Effects of common chemotherapeutic agents used in the treatment of ALL}

\begin{tabular}{|ll|}
\hline Agent & Effects \\
\hline Asparaginase & Hypersensitivity reactions, pancreatitis, thrombosis \\
Clofarabine & $\begin{array}{l}\text { Cardiotoxicity, cytokine release syndrome, hepatotoxicity (including } \\
\text { sinusoidal obstruction syndrome), pancreatitis, nephrotoxicity }\end{array}$ \\
Corticosteroids & Hypertension, hyperglycemia, osteonecrosis, fluid retention, psychosis \\
Cyclophosphamide & Nephrotoxicity, hemorrhagic cystitis, hyponatremia, fluid retention \\
Cytarabine & Conjunctivitis, flu-like symptoms \\
Doxorubicin/daunorubicin & Cardiotoxicity, benign red urine \\
Etoposide & Nephrotoxicity, hepatotoxicity, hypersensitivity reactions \\
Mercaptopurine & Hepatotoxicity \\
Methotrexate & Mucositis, nephrotoxicity, hepatotoxicity, encephalopathy \\
Thioguanine & Hepatotoxicity (including sinusoidal obstruction syndrome and portal \\
hypertension)
\end{tabular}

\section{SUMMARY}

Acute lymphoblastic leukemia is treated with a combination of chemotherapy drugs over the course of several years, with an overall survival of approximately $80 \%$ for all newly diagnosed patients. Those patients with higher risk of relapse receive more aggressive treatment, whereas those with more favorable features can be spared the more toxic effects. Treatment is progressively less intensive as the duration of therapy progresses, and must include central nervous system (CNS) directed therapy regardless of involvement of the CNS at diagnosis. Multi-center randomized clinical trials through international cooperative groups help to further improve survival through the investigation of novel therapeutic approaches.

\section{References}

1. Gaynon PS, Angiolillo AL, Carroll WL, et al. Long-term results of the children's cancer group studies for childhood acute lymphoblastic leukemia 1983-2002: a Children's Oncology Group Report. Leukemia. 2010; 24(2):285-97. [PubMed: 20016531]

2. Möricke A, Zimmermann M, Reiter A, et al. Prognostic impact of age in children and adolescents with acute lymphoblastic leukemia: data from the trials ALL-BFM 86, 90, and 95. Klin Padiatr. 2005; 217(6):310-20. [PubMed: 16307416]

3. Reiter A, Schrappe M, Ludwig WD, et al. Chemotherapy in 998 unselected childhood acute lymphoblastic leukemia patients. Results and conclusions of the multicenter trial ALL-BFM 86. Blood. 1994; 84:3122-33. [PubMed: 7949185]

4. Haydu JE, Ferrando AA. Early T-cell precursor acute lymphoblastic leukaemia. Curr Opin Hematol. 2013; 20(4):369-73. [PubMed: 23695450]

5. Gerr H, Zimmermann M, Schrappe M, et al. Acute leukaemias of ambiguous lineage in children: characterization, prognosis and therapy recommendations. Br J Haematol. 2010; 149(1):84-92. [PubMed: 20085575] 
6. Harris MB, Shuster JJ, Carroll A, et al. Trisomy of leukemic cell chromosomes 4 and 10 identifies children with B-progenitor cell acute lymphoblastic leukemia with a very low risk of treatment failure: a Pediatric Oncology Group study. Blood. 1992; 79(12):3316-24. [PubMed: 1596572]

7. Seeger K, Stackelberg AV, Taube T, et al. Relapse of TEL-AML1-positive acute lymphoblastic leukemia in childhood: a matched-pair analysis. J Clin Oncol. 2001; 19(13):3188-93. [PubMed: 11432885]

8. Pollock BH, DeBaun MR, Camitta BM, et al. Racial differences in the survival of childhood Bprecursor acute lymphoblastic leukemia: a Pediatric Oncology Group Study. J Clin Oncol. 2000; 18(4):813-23. [PubMed: 10673523]

9. Rubnitz JE, Wichlan D, Devidas M, et al. Children's Oncology Group. Prospective analysis of TEL gene rearrangements in childhood acute lymphoblastic leukemia: a Children's Oncology Group study. J Clin Oncol. 2008; 26(13):2186-91. [PubMed: 18445843]

10. Heerema NA, Carroll AJ, Devidas M, et al. Intrachromosomal amplification of chromosome 21 is associated with inferior outcomes in children with acute lymphoblastic leukemia treated in contemporary standard-risk children's oncology group studies: a report from the children's oncology group. J Clin Oncol. 2013; 31(27):3397-402. [PubMed: 23940221]

11. Schrappe M, Hunger SP, Pui CH, et al. Outcomes after induction failure in childhood acute lymphoblastic leukemia. N Engl J Med. 2012; 366(15):1371-81. [PubMed: 22494120]

12. Borowitz MJ, Devidas M, Hunger SP, et al. Children's Oncology Group. Clinical significance of minimal residual disease in childhood acute lymphoblastic leukemia and its relationship to other prognostic factors: a Children's Oncology Group study. Blood. 2008; 111(12):5477-85. [PubMed: 18388178]

13. Conter V, Bartram CR, Valsecchi MG, et al. Molecular response to treatment redefines all prognostic factors in children and adolescents with B-cell precursor acute lymphoblastic leukemia: results in 3184 patients of the AIEOP-BFM ALL 2000 study. Blood. 2010; 115(16):3206-14. [PubMed: 20154213]

14. Escherich G, Zimmermann M, Janka-Schaub G, et al. Doxorubicin or daunorubicin given upfront in a therapeutic window are equally effective in children with newly diagnosed acute lymphoblastic leukemia. A randomized comparison in trial CoALL 07-03. Pediatr Blood Cancer. 2013; 60(2):254-7. [PubMed: 22948968]

15. Mitchell CD, Richards SM, Kinsey SE, et al. Benefit of dexamethasone compared with prednisolone for childhood acute lymphoblastic leukaemia: results of the UK Medical Research Council ALL97 randomized trial. Br J Haematol. 2005; 129(6):734-45. [PubMed: 15952999]

16. Avramis VI, Sencer S, Periclou AP, et al. A randomized comparison of native Escherichia coli asparaginase and polyethylene glycol conjugated asparaginase for treatment of children with newly diagnosed standard-risk acute lymphoblastic leukemia: a Children's Cancer Group study. Blood. 2002; 99(6):1986-94. [PubMed: 11877270]

17. Seibel NL, Steinherz PG, Sather HN, et al. Early postinduction intensification therapy improves survival for children and adolescents with high-risk acute lymphoblastic leukemia: a report from the Children's Oncology Group. Blood. 2008; 111(5):2548. [PubMed: 18039957]

18. Bhatia S, Landier W, Shangguan M, et al. Nonadherence to oral mercaptopurine and risk of relapse in Hispanic and non-Hispanic white children with acute lymphoblastic leukemia: a report from the children's oncology group. J Clin Oncol. 2012; 30(17):2094-101. [PubMed: 22564992]

19. Brackett J, Schafer ES, Leung DH, et al. Use of allopurinol in children with acute lymphoblastic leukemia to reduce skewed thiopurine metabolism. Pediatr Blood Cancer. 2014; 61(6):1114-7. [PubMed: 24376133]

20. Schmiegelow K, Schrøder H, Gustafsson G, et al. Risk of relapse in childhood acute lymphoblastic leukemia is related to RBC methotrexate and mercaptopurine metabolites during maintenance chemotherapy. Nordic Society for Pediatric Hematology and Oncology. J Clin Oncol. 1995; 13(2): 345-51. [PubMed: 7531219]

21. Schmiegelow K, Heyman M, Gustafsson G, et al. The degree of myelosuppression during maintenance therapy of adolescents with B-lineage intermediate risk acute lymphoblastic leukemia predicts risk of relapse. Leukemia. 2010; 24(4):715-20. [PubMed: 20130603] 
22. Eden TO, Pieters R, Richards S, et al. Systematic review of the addition of vincristine plus steroid pulses in maintenance treatment for childhood acute lymphoblastic leukaemia - an individual patient data meta-analysis involving 5,659 children. Br J Haematol. 2010; 149(5):722-33. [PubMed: 20331462]

23. Evans AE, Gilbert ES, Zandstra R. The increasing incidence of central nervous system leukemia in children. (Children's Cancer Study Group A). Cancer. 1970; 26:404. [PubMed: 5271211]

24. Matloub Y, Lindemulder S, Gaynon PS, et al. Intrathecal triple therapy decreases central nervous system relapse but fails to improve event-free survival when compared with intrathecal methotrexate: results of the Children's Cancer Group (CCG) 1952 study for standard-risk acute lymphoblastic leukemia, reported by the Children's Oncology Group. Blood. 2006; 108(4):116573. [PubMed: 16609069]

25. Pui CH, Campana D, Pei D, et al. Treating childhood acute lymphoblastic leukemia without cranial irradiation. N Engl J Med. 2009; 360(26):2730-41. [PubMed: 19553647]

26. Sirvent N, Suciu S, Rialland X, et al. Prognostic significance of the initial cerebrospinal fluid (CSF) involvement of children with acute lymphoblastic leukaemia (ALL) treated without cranial irradiation: results of European Organization for Research and Treatment of Cancer (EORTC) Children Leukemia Group study 58881. Eur J Cancer. 2011; 47(2):239-47. [PubMed: 21095115]

27. Hochberg J, Khaled S, Forman SJ, et al. Criteria for and outcomes of allogeneic haematopoietic stem cell transplant in children, adolescents and young adults with acute lymphoblastic leukaemia in first complete remission. Br J Haematol. 2013; 161(1):27-42. [PubMed: 23384118]

28. Hilden JM, Dinndorf PA, Meerbaum SO, et al. Analysis of prognostic factors of acute lymphoblastic leukemia in infants: report on CCG 1953 from the Children's Oncology Group. Blood. 2006; 108(2):441-51. [PubMed: 16556894]

29. Pieters R, Schrappe M, De Lorenzo P, et al. A treatment protocol for infants younger than 1 year with acute lymphoblastic leukaemia (Interfant-99): an observational study and a multicentre randomised trial. Lancet. 2007; 370(9583):240-50. [PubMed: 17658395]

30. Pichler H, Reismüller B, Steiner M, et al. The inferior prognosis of adolescents with acute lymphoblastic leukaemia (ALL) is caused by a higher rate of treatment-related mortality and not an increased relapse rate-a population-based analysis of 25 years of the Austrian ALL-BFM (Berlin-Frankfurt-Münster) Study Group. Br J Haematol. 2013; 161(4):556-65. [PubMed: 23480776]

31. O'Brien SH, Klima J, Termuhlen AM, et al. Venous thromboembolism and adolescent and young adult oncology inpatients in US children's hospitals, 2001 to 2008. J Pediatr. 2011; 159(1):133-7. [PubMed: 21353248]

32. Ram R, Wolach O, Vidal L, et al. Adolescents and young adults with acute lymphoblastic leukemia have a better outcome when treated with pediatric-inspired regimens: systematic review and meta-analysis. Am J Hematol. 2012; 87(5):472-8. [PubMed: 22388572]

33. Berg SL, Blaney SM, Devidas M, et al. Phase II study of nelarabine (compound 506U78) in children and young adults with refractory T-cell malignancies: A report from the Children's Oncology Group. J Clin Oncol. 2005; 15:3376-82. [PubMed: 15908649]

34. Rubnitz JE, Onciu M, Pounds S, et al. Acute mixed lineage leukemia in children: the experience of St Jude Children's Research Hospital. Blood. 2009; 113(21):5083-9. [PubMed: 19131545]

35. Whitlock JA. Down syndrome and acute lymphoblastic leukaemia. Br J Haematol. 2006; 135(5): 595-602. [PubMed: 17054672]

36. Schultz KR, Bowman WP, Aledo A, et al. Improved early event-free survival with imatinib in Philadelphia chromosome-positive acute lymphoblastic leukemia: a children's oncology group study. J Clin Oncol. 2009; 27(31):5175-81. [PubMed: 19805687]

37. Locatelli F, Schrappe M, Bernardo ME, et al. How I treat relapsed childhood acute lymphoblastic leukemia. Blood. 2012; 120(14):2807-16. [PubMed: 22896001]

38. Chessells JM. Relapsed lymphoblastic leukaemia in children: a continuing challenge. $\mathrm{Br}$ J Haematol. 1998; 102(2):423-38. [PubMed: 9695956]

39. Nguyen K, Devidas M, Cheng SC, et al. Factors influencing survival after relapse from acute lymphoblastic leukemia: a Children's Oncology Group study. Leukemia. 2008; 22(12):2142-50. [PubMed: 18818707] 
40. Coustan-Smith E, Gajjar A, Hijiya N, et al. Clinical significance of minimal residual disease in childhood acute lymphoblastic leukemia after first relapse. Leukemia. 2004; 18(3):499-504. [PubMed: 14981525]

41. Eckert C, von Stackelberg A, Seeger K, et al. Minimal residual disease after induction is the strongest predictor of prognosis in intermediate risk relapsed acute lymphoblastic leukaemia long-term results of trial ALL-REZ BFM P95/96. Eur J Cancer. 2013; 49(6):1346-55. [PubMed: 23265714]

42. Gandemer V, Chevret S, Petit A, et al. Excellent prognosis of late relapses of ETV6/RUNX1positive childhood acute lymphoblastic leukemia: lessons from the FRALLE 93 protocol. Haematologica. 2012; 97(11):1743-50. [PubMed: 22580999]

43. Hof J, Krentz S, van Schewick C, et al. Mutations and deletions of the TP53 gene predict nonresponse to treatment and poor outcome in first relapse of childhood acute lymphoblastic leukemia. J Clin Oncol. 2011; 29(23):3185-93. [PubMed: 21747090]

44. Parker C, Waters R, Leighton C, et al. Effect of mitoxantrone on outcome of children with first relapse of acute lymphoblastic leukaemia (ALL R3): an open-label randomised trial. Lancet. 2010; 376(9757):2009-17. [PubMed: 21131038]

45. Rivera GK, Hudson MM, Liu Q, et al. Effectiveness of intensified rotational combination chemotherapy for late hematologic relapse of childhood acute lymphoblastic leukemia. Blood. 1996; 88(3):831-7. [PubMed: 8704238]

46. Wofford MM, Smith SD, Shuster JJ, et al. Treatment of occult or late overt testicular relapse in children with acute lymphoblastic leukemia: a Pediatric Oncology Group study. J Clin Oncol. 1992; 10(4):624-30. [PubMed: 1548525]

47. Levine JE, Barrett AJ, Zhang MJ, et al. Donor leukocyte infusions to treat hematologic malignancy relapse following allo-SCT in a pediatric population. Bone Marrow Transplant. 2008; 42(3):2015. [PubMed: 18490913]

48. Brentjens RJ, Curran KJ. Novel cellular therapies for leukemia: CAR-modified T cells targeted to the CD19 antigen. Hematology Am Soc Hematol Educ Program. 2012; 2012:143-51. [PubMed: 23233573]

49. Hoffman LM, Gore L. Blinatumomab, a Bi-Specific Anti-CD19/CD3 BiTE(®) antibody for the treatment of acute lymphoblastic leukemia: perspectives and current pediatric applications. Front Oncol. 2014; 4:63. [PubMed: 24744989]

50. Kreitman RJ, Pastan I. Antibody fusion proteins: anti-CD22 recombinant immunotoxin moxetumomab pasudotox. Clin Cancer Res. 2011; 17(20):6398-405. [PubMed: 22003067]

51. Burke MJ, Bhatla T. Epigenetic modifications in pediatric acute lymphoblastic leukemia. Front Pediatr. 2014; 2:42. [PubMed: 24860797]

52. Pui CH, Jeha S. New therapeutic strategies for the treatment of acute lymphoblastic leukaemia. Nat Rev Drug Discov. 2007; 6:149-65. [PubMed: 17268486] 


\section{KEY POINTS}

- Pediatric acute lymphoblastic leukemia is the most common cancer diagnosed in children.

- Risk stratification allows treatment intensity to vary based on risk of treatment failure, and is based on age, initial leukocyte count, involvement of sanctuary sites, immunophenotype, cytogenetics, and response to treatment.

- The 4 main components of therapy are remission induction, consolidation, maintenance, and central nervous system-directed therapy, with an overall duration of therapy of 2 to 3 years.

- Prognosis after relapse depends on site of relapse and duration of initial remission. 


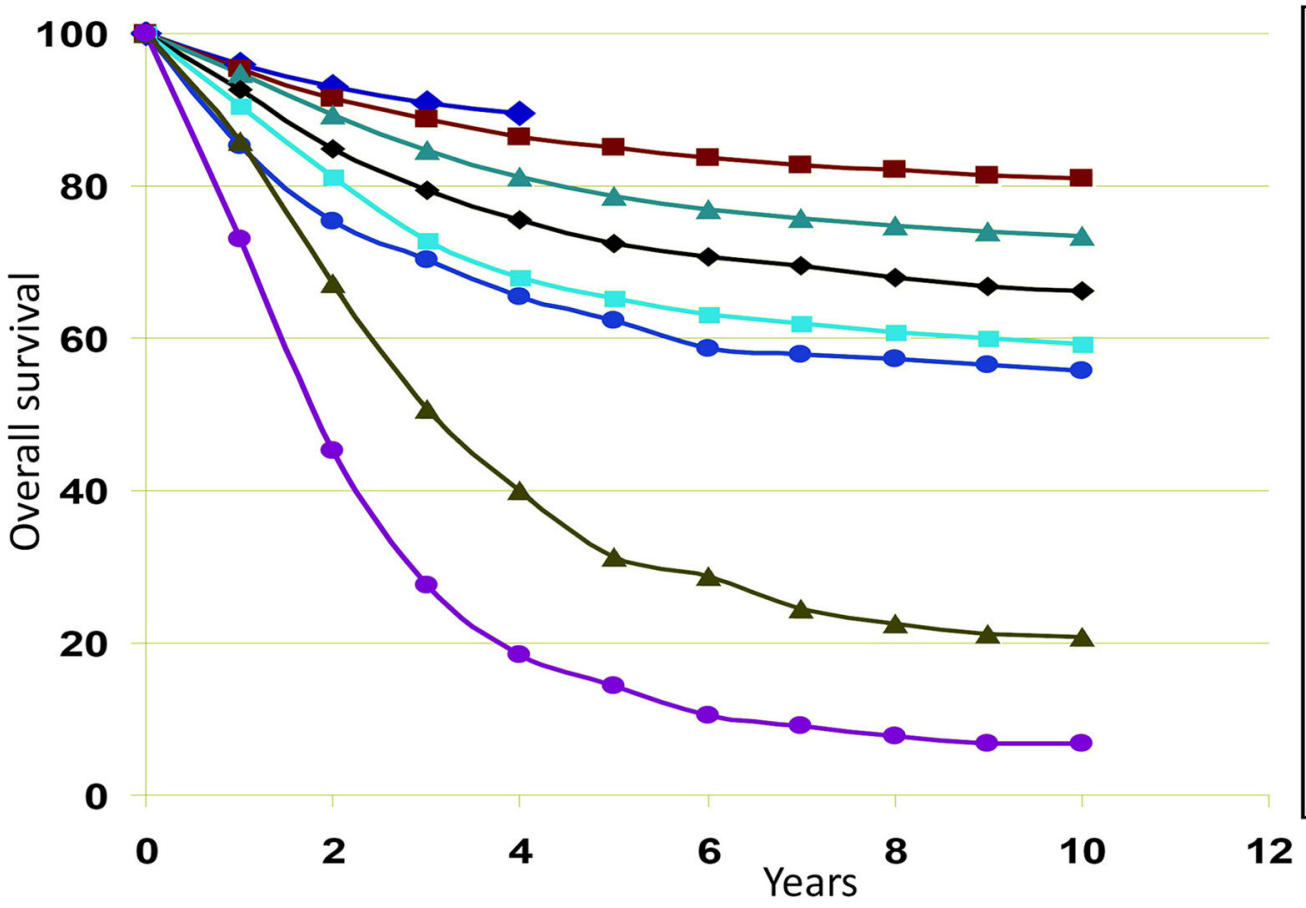

$\checkmark$ 1996-2000 $(n=3421)$

$\rightarrow-1989-1995$ $(n=5121)$

$-1983-1988$ $(n=3711)$

$\multimap$ 1978-1983 ( $n=2984)$

$-1975-1977$ $(n=1313)$

$\multimap$ 1972-1975 $(n=936)$

$-1970-1972$ $(n=499)$

$\longrightarrow$ 1968-1970 $(n=402)$

Fig. 1.

Improved overall survival in childhood acute lymphoblastic leukemia (ALL). (From Hunger SP, Winick NJ, Sather HN et al. Therapy of low-risk subsets of childhood acute lymphoblastic leukemia: when do we say enough? Pediatr Blood Cancer 2005;45(7):87680 ; with permission.) 


\section{Table 1}

Outcomes for newly diagnosed childhood acute lymphoblastic leukemia

\begin{tabular}{|lllll|}
\hline Cooperative Group & Study & Years & Patients & 5-y EFS (\%) \\
\hline Berlin-Frankfurt-Münster $^{52}$ & ALL-BFM-95 & $1995-2000$ & 2169 & $79.6^{a}$ \\
\hline Children's Oncology Group $^{52}$ & Multiple & $2000-2005$ & 7153 & 90.4 \\
\hline Dana Farber Cancer Institute Consortium $^{52}$ & DFCI 95-01 & $1996-2001$ & 491 & 82.0 \\
\hline Nordic Society of Pediatric Hematology and Oncology $^{52}$ & NOPHO & $2002-2007$ & 1023 & 79.0 \\
\hline St Jude Children's Research Hospital $^{52}$ & TOTXV & $2000-2007$ & 498 & 85.6 \\
\hline United Kingdom Acute Lymphoblastic Leukaemia $^{52}$ & UKALL 2003 & $2003-2011$ & 3126 & 87.2 \\
\hline
\end{tabular}

Abbreviation: EFS, event-free survival.

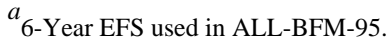

